Published in final edited form as:

Curr Opin Immunol. 2009 June ; 21(3): 346-351. doi:10.1016/j.coi.2009.05.016.

\title{
Heterologous Prime-Boost Vaccination
}

\author{
Shan Lu \\ China-US Vaccine Research Center and Department of Infectious Diseases, The First Affiliated \\ Hospital, Nanjing Medical University, Nanjing, 210029, China, \& Department of Medicine, \\ University of Massachusetts Medical School 364 Plantation St., Worcester, 01605, USA
}

Shan Lu: shan.lu@umassmed.edu

\section{Summary}

An effective vaccine usually requires more than one time immunization in the form of primeboost. Traditionally the same vaccines are given multiple times as homologous boosts. New findings suggested that prime-boost can be done with different types of vaccines containing the same antigens. In many cases such heterologous prime-boost can be more immunogenic than homologous prime-boost. Heterologous prime-boost represents a new way of immunization and will stimulate better understanding on the immunological basis of vaccines.

\section{Introduction}

It is not unusual that multiple immunizations are required for many vaccines to be successful. For pediatric population, up to five immunizations may be needed, as is the case for Diphtheria, Tetanus and Pertussis (DTP) vaccine, which is given three times during the first six months after birth, followed by a fourth dose in the second year of life, and a final boost between four and six years of age. Still, some of the vaccines need additional boosts even in adults who have already received the complete immunization series, for example, the Tetanus-diphtheria (Td) vaccine, for which a boost is recommended every 10 years throughout a person's lifespan. While it is not entirely clear why some vaccines require more immunizations than others, it is well accepted that multiple immunizations (i.e. "prime-boost") are critical for even the most successful vaccines. This principle applies to live attenuate vaccines (e.g., oral polio vaccine), inactivated vaccines (e.g., hepatitis A vaccine), recombinant protein subunit vaccines (e.g., hepatitis B vaccine) and polysaccharide vaccines (e.g., Haemophilus Influenzae type b vaccine). For these vaccines, the prime-boost is "homologous" because the same vaccines given in the earlier priming immunizations are used for subsequent boost immunizations.

Over the past decade, studies have shown that prime-boost immunizations can be given with unmatched vaccine delivery methods while using the same antigen, in a "heterologous" prime-boost format. The most interesting and unexpected finding is that, in many cases, heterologous prime-boost is more effective than the "homologous" prime-boost approach. The rapid progress of novel vaccination approaches, such as DNA vaccines and viral vector-

(C) 2009 Elsevier Ltd. All rights reserved.

Correspondence to: Shan Lu, shan. lu@umassmed. edu.

Publisher's Disclaimer: This is a PDF file of an unedited manuscript that has been accepted for publication. As a service to our customers we are providing this early version of the manuscript. The manuscript will undergo copyediting, typesetting, and review of the resulting proof before it is published in its final citable form. Please note that during the production process errors may be discovered which could affect the content, and all legal disclaimers that apply to the journal pertain. 
based vaccines, has certainly further expanded the scope of heterologous prime-boost vaccination [1-3].

\section{Early history of heterologous prime-boost vaccination}

A 1992 landmark Science report was among the first to employ the heterologous primeboost immunization technique in a non-human primate model [4]. In that study, Macaca fascicularis were first immunized with recombinant vaccinia virus expressing SIVmne gp160 antigen and then boosted with gp160 protein produced in baculovirus-infected cells. Animals were protected from intravenous challenge of SIVmne viruses and this became one of the most promising protection results in the early HIV vaccine development effort.

Shiu-Lok Hu, the lead scientist of the above study, and his collaborators demonstrated previously, in rodents, that priming with a live recombinant virus and boosting with a subunit recombinant protein was more effective than immunization by either immunogen alone [5]. In a separate study, Girard et al. also reported a significant increase in antibody titers in a chimpanzee primed with recombinant vaccinia virus and boosted multiple times with a mixture of recombinant HIV-1 proteins or synthetic peptides [6]. Furthermore, around the same time, in what may be the first human testing of the heterologous primeboost immunization, Daniel Zagury of the Pierre and Marie Curie University in Paris inoculated himself with a recombinant vaccinia virus containing the HIV-1 Env gene and later gave a boost using a recombinant Env protein [7]. Early work in other non-HIV areas include small animal studies conducted by Eckhart Wimmer's group who used synthetic peptides and inactivated polio virus for prime-boost immunizations [8].

\section{Heterologous prime-boost HIV-1 vaccines}

Initial efforts in the use of a heterologous prime-boost immunization approach for HIV-1 vaccine development was based on the following rationale:

Recombinant envelope (Env) glycoproteins, while being able to elicit isolate specific neutralizing antibody responses, were unable to elicit cytotoxic $\mathrm{T}$ cell responses, and on the other hand, immunization with recombinant vaccinia expressing HIV-1 antigens could elicit good $\mathrm{T}$ cell responses but not high levels of protective antibodies. Therefore combined immunization including both of these two types of vaccines may be more effective than either immunogen alone [5].

This statement established a key principle for the use of heterologous prime-boost immunizations, i.e., to elicit both humoral and cell-mediated immune responses. Modern immunology has established that such a balanced immune response is important for protection not only against viral infections but also other types of pathogens. Traditional vaccines, particularly inactivated and subunit vaccines, are not very effective in eliciting $\mathrm{T}$ cell responses. This requirement is even more important for HIV vaccine development. An ideal HIV vaccine should be able to generate "sterilizing antibodies" to prevent the virus from establishing an infection that is more difficult to eliminate once HIV-1 is integrated into the genome of the host's peripheral blood mononuclear cells (PBMCs).

At the same time, $\mathrm{T}$ cell immune responses play a key role in controlling the scale of infection, which may affect the long-term mortality and morbidity of the host.

The discovery of DNA vaccines in early 1990s added a new vaccine modality to the traditional vaccine delivery approaches. After showing promising utility of DNA vaccines, mainly in small animal models, the immunogenicity of DNA vaccines in humans was soon realized too low to be used alone although DNA vaccination proved its worth as an excellent 
priming modality [9]. As a result, DNA priming, along with various forms of boost, has been incorporated into almost every current major HIV-1 vaccine development effort.

Similar to the original vaccinia prime-recombinant protein boost HIV-1 vaccines which used antigens from laboratory adapted HIV-1 isolates, protein boost can also be given after the DNA prime [10,11]. Actually, DNA prime and recombinant protein boost with primary HIV-1 Env antigens became the first approach to elicit positive neutralizing antibodies in rabbit sera against JR-FL, a fairly difficult to neutralize primary HIV-1 isolate [12, 13]. The same prime-boost approach can be used to deliver multiple Env antigens that can either be matched or not matched between the DNA prime and protein boost. Such polyvalent Env formulations were more likely to elicit cross-reactive neutralizing antibodies against a broad spectrum of primary HIV-1 viruses than the monovalent Env formulations [14]. The DNA prime-protein boost approach was also proven effective in non-human primates [15] and more significantly, in humans [16]. In these studies, four out of six rhesus macaques immunized with DNA prime-protein boost were able to achieve "sterilizing" protection [15]. In human volunteers, no antigen-specific antibody responses were detected after three DNA immunizations, but a quick rise of anti-Env IgG responses to titers of 1:104 to 1:105 were identified after only one protein boost in every volunteer that received this combination vaccine. These human immune sera were cross-reactive to Env antigens from every clade of HIV-1 isolates with positive neutralizing activities against primary HIV-1 from clades A to E [16]. This result is particularly striking as no other candidate HIV vaccine has been able to induce such a response in humans. More significantly, this DNA prime-protein boost formation also elicited high frequency of responders with HIV-1 antigen specific and polyfunctional $\mathrm{T}$ cell immune responses [16, 17].

Due to the failure of the VaxGen HIV vaccine trial, which only used recombinant gp120 proteins as immunogens, T cell-based vaccines have been the main focus in HIV vaccine development for the past decade. Due to safety concerns over the use of live attenuated HIV-1 vaccines, there are limited vaccine platforms to elicit high level T cell immune responses. Novel vaccine delivery approaches, such as DNA and viral vector-based vaccines, have become the main weapons to elicit $\mathrm{T}$ cell-mediated immune responses, particularly when these two approaches are delivered as a prime-boost. Results from a number of non-human primate studies have shown good immunogenicity and protection against chimeric SHIV challenge using the DNA prime-viral vector boost combination. Viral vector boosts have included MVA vector [18, 19], fowlpox vector [20], adenoviral vectors [21, 22] and Vesicular Stomatitis Virus (VSV) vector [23]. Some DNA prime-viral vector boost HIV-1 vaccines have also moved into human studies with clearly positive antigen-specific $\mathrm{T}$ cell immune responses. The Euro Vacc 02 phase I clinical trial provided the clear evidence in a comparative study that a DNA prime-NYVAC poxviral vector boost HIV-1 vaccine was more immunogenic than the NYVAC poxviral vector alone vaccine [24].

Using the similar strategy reported in early 1990s [4, 5], the ALVAC vector prime-protein boost HIV vaccine has been tested in an ongoing phase III clinical trial in Thailand. According to the phase $1 / 2$ report, this combination HIV-1 vaccine was shown to be immunogenic as evidenced by positive antigen-specific antibody and positive CD8+ T cell responses [25]. While it is not clear whether this particular formulation will lead to a successful protective HIV-1 vaccine due to the selection of Env antigens that may have very limited cross-reactivity, the combination approach itself has proven successful in improving the immunogencity of otherwise two relatively weak vaccine components. 


\section{Heterologous prime-boost vaccines against other pathogens}

Over the past few years, the use of heterologous prime-boost approaches in vaccine research has gained significant momentum against a wide range of pathogens. Several features have become apparent for this trend.

First, it is common to use the heterologous prime-boost approach to address some of the most challenging vaccine development objectives including malaria and tuberculosis (for detailed information please see accompanying articles in this volume) due to the failure of other vaccination approaches. The idea is to focus on certain critical antigens and to elicit high quality immune responses involving different subsets of $\mathrm{T}$ cell immune responses. A DNA prime-MVA boost vaccine encoding thrombospondin-related adhesion protein partially protected healthy malaria-naïve adults against Plasmodium falciparum sporozoite challenge [26]. This study also highlights the importance of antigen selection for immune protection, made clear by the fact that the same combination vaccination using circumsporozoite protein, instead of the thrombospondin-related adhesion protein, did not elicit such protection.

For tuberculosis vaccine development, qualitatively and quantitatively different cellular immune responses have been elicited in rhesus macaques receiving a recombinant Bacille Calmette-Guérin (BCG) prime followed by an adenovirus 35 vector boost that expressed a fusion protein composed of Ag85A, Ag85B and TB104 [27]. Alternatively, BCG can be used as a boost following a DNA vaccine prime. In one study conducted in calves, DNA prime with Ag85B, MPT64 and MPT83 antigens followed by a BCG boost was able to elicit higher immune responses and better protection than BCG alone against Mycobacterium bovis challenge [28].

Second, a well-designed heterologus prime-boost approach can expand the scope of immune responses. When mice were primed with DNA vaccine expressing ESAT6 and later received the same antigen in the form of recombinant protein as boost, production of Th1-type cytokines was increased significantly, as was the $\mathrm{IgG} 2$ to $\mathrm{IgG} 1$ ratio [29]. In another murine study, prime with a DNA vaccine, expressing the $\mathrm{gD}$ antigen of herpes simplex virus type 2 (HSV-2), which preferentially induces Th1 type cellular immune responses, and boost with recombinant $\mathrm{gD}$ protein, which mainly induces $\mathrm{Th} 2$ biased responses, led to significantly enhanced antibody, T cell proliferation, and Th1 cytokine production [30].

Third, the prime-boost vaccine approach can also improve the effectiveness of existing vaccines. One example is the use of DNA prime, which increased antibody response levels, in animals later receiving boost with inactivated rabies vaccines [31]. Similarly, DNA prime can increase the titer and longevity of hyperimmune sera in animals to be immunized with the recombinant PA antigen against anthrax [32]. Adding a DNA prime, mice boosted with the licensed hepatitis B surface protein vaccine were able to produce stronger and more homogenous antibody responses in a study group when compare to groups only receiving recombinant protein alone. Higher IL-12 and IFN- $\gamma$ secretion in splenocytes were also observed [33].

Finally, the prime-boost approach can have important practical applications in addressing vaccines with broad public health impact. In an animal model naïve to influenza infection, it has been shown that a heterologous one-time DNA prime and one-time inactivated influenza vaccine boost was more immunogenic than twice administered homologous prime-boost using either DNA or inactivated influenza vaccine alone [34]. This finding can be very useful for preparation against pandemic avian influenza. One of the key issues facing the development of influenza vaccines is the limited capacity and long cycle needed to produce traditional influenza vaccines. Usually two immunizations are needed for avian influenza 
vaccines. It is feasible that targeted populations can first receive an avian influenza DNA vaccine prime long before any unexpected pandemic attack, which will greatly reduce the amount of vaccine needed at the time of outbreak of pandemic flu. This approach can also be useful for other forms of influenza, including human and swine influenza viruses. Adding a new strain of vaccine to the current trivalent influenza vaccines will require significant additional resources and time. A polyvalent DNA prime can cover a wide range of future potential viral strains at much lower cost.

\section{Heterologous prime-boost vaccines as therapy to cancers}

Similar to other novel vaccine forms, the heterologous prime-boost approaches have also been studied as potential treatments for cancer. Using a recently identified sixtransmembrane epithelial antigen of the prostate (STEAP), a heterologous DNA prime and Venezuelan equine encephalitis virus-like replicon particles (VRP) boost was able to elicit better immune responses against STEAP, including INF-gamma, TNF-alpha, and IL-12, when compared to either vaccine modality alone. This vaccination regimen induced a modest but significant delay in growth of established, 31 day-old tumors in mice [35].

\section{Mechanisms of heterologous prime-boost vaccines}

A fundamental but still mysterious question is why the heterlogous prime-boost is more effective than homologous prime-boost even when the same vaccine components are used for each. One way to study this question is to determine the importance of order of administration of heterologous prime-boost vaccines. Using a Mycobacterium bovis model, it was demonstrated that the order of prime-boost vaccination of neonatal calves with BCG and DNA vaccine, encoding Hsp65, Hsp70 and Apa, was not critical for enhancing protection against bovine tuberculosis [36]. In a different model, with DNA prime-protein boost using murine HSV-2 gD antigen, it was clear that DNA priming is critical because a reversed protein prime-DNA boost regimen produced antibody levels similar to those following homologous protein-protein vaccination, and failed to further enhance Th cell proliferative responses or cytokine production [30]. In an even more detailed analysis using hepatitis C E2 as a model antigen, it was found that DNA prime-adenoviral vector boost elicited the highest level of Th1 CD4+ T cell responses when compared to the reversed adenoviral prime-DNA boost or homologous prime-boost with the same vaccines. More interestingly, the DNA prime-adenoviral vector boost regimen, but none of the other three possible prime-boost combinations, elicited CTL responses against three E2-specific epitopes and one of them was immunodominant [37].

In an extensive non-human primate study, presented at the 2008 AIDS Vaccine conference in Cape Town, South Africa by Dr. Shiu-lok Hu from University of Washington, Seattle, vaccinia viral vector or DNA prime, followed by protein boost, generated better antibody responses than boosting with DNA or various viral vector vaccines. These two heterologous prime-boost regimens, including a protein boost component, but not any of the other combinations, were able to elicit better neutralizing antibodies and sterilizing immunity against a high-dose intrarectal challenge by $\mathrm{SHIV}_{\text {sf162.p } 4}$ in $\sim 40 \%$ of immunized animals, and protected animals against peripheral CD4+ T-cell depletion.

Some studies have shown that DNA prime was able to improve the avidity of antibody responses elicited by protein-based vaccines $[11,13]$. Because DNA vaccines produce antigens in vivo, priming with a DNA vaccine may elicit memory B cells that are specific to sensitive conformation domains of an antigen. In a rabbit study, the delivery of primary HIV-1 gp120 antigens using the DNA prime-protein boost approach, but not the recombinant gp120 protein alone vaccine, was able to elicit conformation dependent CD4 binding site antibodies which are potentially important for neutralizing HIV-1 [38]. 
The immunogenicity of heterologous prime-boost can be further improved by including other factors that may further facilitate or enhance the effect of vaccines. For example, including plasmid cytokines and colony-stimulating factors could enhance the immunogenicity of DNA prime-viral vector boosting HIV-1 vaccines [22]. The potency of DNA vaccine prime can be enhanced by using a micorparticle based formulation followed with a protein boost [39]. However, it is not clear whether using different adjuvants for a protein vaccine as boost will make any difference.

\section{Conclusions}

Heterologous prime-boost vaccination, using both traditional and novel immunization approaches, provides exciting opportunities to elicit unique immune responses to allow for improved immunogenicity and/or protection. Research has shown that the heterologous prime-boost can take various forms and that the order of prime-boost administration may be important although this may be antigen-dependent and may be influenced by the host species and the type(s) of immune responses to be achieved. Future studies will need to focus more on the mechanisms behind the heterlogous prime-boost vaccination approach and solve practical issues related to a two-component vaccine, including costs of vaccines and any currently unidentified issues of safety.

\section{Acknowledgments}

This work is supported in part by NIH/NIAID grant R01 AI06250. Author would like to thank Michael Vaine, Shiu-lok Hu and Stanley Plotkin for discussion and support in providing related references.

\section{References}

1. Excler JL, Plotkin S. The prime-boost concept applied to HIV preventive vaccines. Aids. 1997; 11(Suppl A):S127-S137. [PubMed: 9451976]

2. Ramshaw IA, Ramsay AJ. The prime-boost strategy: exciting prospects for improved vaccination. Immunol Today. 2000; 21:163-165. [PubMed: 10740236]

3. Lu S. Combination DNA plus protein HIV vaccines. Springer Semin Immunopathol. 2006; 28:255265. [PubMed: 17021720]

4. Hu SL, Abrams K, Barber GN, Moran P, Zarling JM, Langlois AJ, Kuller L, Morton WR, Benveniste RE. Protection of macaques against SIV infection by subunit vaccines of SIV envelope glycoprotein gp160. Science. 1992; 255:456-459. [PubMed: 1531159] • First major report on the use of heterologous prime-boost vaccination approach, in the context of AIDS vaccine development.

5. Hu SL, Klaniecki J, Dykers T, Sridhar P, Travis BM. Neutralizing antibodies against HIV-1 BRU and SF2 isolates generated in mice immunized with recombinant vaccinia virus expressing HIV-1 (BRU) envelope glycoproteins and boosted with homologous gp160. AIDS Res Hum Retroviruses. 1991; 7:615-620. [PubMed: 1768463]

6. Girard M, Kieny MP, Pinter A, Barre-Sinoussi F, Nara P, Kolbe H, Kusumi K, Chaput A, Reinhart $\mathrm{T}$, Muchmore E, et al. Immunization of chimpanzees confers protection against challenge with human immunodeficiency virus. Proc Natl Acad Sci U S A. 1991; 88:542-546. [PubMed: 1988952]

7. Zagury D, Bernard J, Cheynier R, Desportes I, Leonard R, Fouchard M, Reveil B, Ittele D, Lurhuma $\mathrm{Z}$, Mbayo K, et al. A group specific anamnestic immune reaction against HIV-1 induced by a candidate vaccine against AIDS. Nature. 1988; 332:728-731. [PubMed: 3162762]

8. Emini EA, Jameson BA, Wimmer E. Priming for and induction of anti-poliovirus neutralizing antibodies by synthetic peptides. Nature. 1983; 304:699-703. [PubMed: 6310403]

9. Lu S, Wang S, Grimes-Serrano JM. Current progress of DNA vaccine studies in humans. Expert Rev Vaccines. 2008; 7:175-191. [PubMed: 18324888] 
10. Barnett SW, Rajasekar S, Legg H, Doe B, Fuller DH, Haynes JR, Walker CM, Steimer KS. Vaccination with HIV-1 gp120 DNA induces immune responses that are boosted by a recombinant gp120 protein subunit. Vaccine. 1997; 15:869-873. [PubMed: 9234536]

11. Richmond JF, Lu S, Santoro JC, Weng J, Hu SL, Montefiori DC, Robinson HL. Studies of the neutralizing activity and avidity of anti-human immunodeficiency virus type 1 Env antibody elicited by DNA priming and protein boosting. J Virol. 1998; 72:9092-9100. [PubMed: 9765454]

12. Beddows S, Schulke N, Kirschner M, Barnes K, Franti M, Michael E, Ketas T, Sanders RW, Maddon PJ, Olson WC, et al. Evaluating the immunogenicity of a disulfide-stabilized, cleaved, trimeric form of the envelope glycoprotein complex of human immunodeficiency virus type 1 . $\mathrm{J}$ Virol. 2005; 79:8812-8827. [PubMed: 15994775]

13. Wang S, Arthos J, Lawrence JM, Van Ryk D, Mboudjeka I, Shen S, Chou TH, Montefiori DC, Lu S. Enhanced immunogenicity of gp120 protein when combined with recombinant DNA priming to generate antibodies that neutralize the JR-FL primary isolate of human immunodeficiency virus type 1. J Virol. 2005; 79:7933-7937. [PubMed: 15919951]

14. Wang S, Pal R, Mascola JR, Chou TH, Mboudjeka I, Shen S, Liu Q, Whitney S, Keen T, Nair BC, et al. Polyvalent HIV-1 Env vaccine formulations delivered by the DNA priming plus protein boosting approach are effective in generating neutralizing antibodies against primary human immunodeficiency virus type 1 isolates from subtypes A, B, C, D and E. Virology. 2006; 350:3447. [PubMed: 16616287] - By using DNA prime-protein boost vaccination to deliver polyvalent HIV-1 Env antigens, broadly reactive neutralizing antibodies are elicited immunized sera in a rabbit model.

15. Pal R, Kalyanaraman VS, Nair BC, Whitney S, Keen T, Hocker L, Hudacik L, Rose N, Mboudjeka I, Shen S, et al. Immunization of rhesus macaques with a polyvalent DNA prime/protein boost human immunodeficiency virus type 1 vaccine elicits protective antibody response against simian human immunodeficiency virus of R5 phenotype. Virology. 2006; 348:341-353. [PubMed: 16460776]

16. Wang S, Kennedy JS, West K, Montefiori DC, Coley S, Lawrence J, Shen S, Green S, Rothman AL, Ennis FA, et al. Cross-subtype antibody and cellular immune responses induced by a polyvalent DNA prime-protein boost HIV-1 vaccine in healthy human volunteers. Vaccine. 2008; 26:1098-1110. [PubMed: 18243434] •• First report of inducing broadly reactive NAb in humans in addition to high level cell mediated immune responses using a polyvalent, multi-gene DNA prime-protein boost HIV vaccine.

17. Bansal A, Jackson B, West K, Wang S, Lu S, Kennedy JS, Goepfert PA. Multifunctional T-cell characteristics induced by a polyvalent DNA prime/protein boost human immunodeficiency virus type 1 vaccine regimen given to healthy adults are dependent on the route and dose of administration. J Virol. 2008; 82:6458-6469. [PubMed: 18448544] • When a DNA prime-protein boost HIV vaccine was tested in humans, the DNA priming effect delivered by intramuscular (IM) needle injection was more effective than the intradermal (ID) needle injection in eliciting HIV-1 antigen specific cell mediated immune responses, which is contrary to the long-held hypothesis that ID route is more immunogenic than IM route for DNA vaccines.

18. Amara RR, Villinger F, Altman JD, Lydy SL, O'Neil SP, Staprans SI, Montefiori DC, Xu Y, Herndon JG, Wyatt LS, et al. Control of a mucosal challenge and prevention of AIDS by a multiprotein DNA/MVA vaccine. Science. 2001; 292:69-74. [PubMed: 11393868] - Supported by Shiver et al. [21], this report showed that DNA prime-MVA viral vector boost approach was able to elicit protective immune response against SHIV in a non-human primate model.

19. Hanke T, Samuel RV, Blanchard TJ, Neumann VC, Allen TM, Boyson JE, Sharpe SA, Cook N, Smith GL, Watkins DI, et al. Effective induction of simian immunodeficiency virus-specific cytotoxic T lymphocytes in macaques by using a multiepitope gene and DNA prime-modified vaccinia virus Ankara boost vaccination regimen. J Virol. 1999; 73:7524-7532. [PubMed: 10438842]

20. Kent SJ, Zhao A, Best SJ, Chandler JD, Boyle DB, Ramshaw IA. Enhanced T-cell immunogenicity and protective efficacy of a human immunodeficiency virus type 1 vaccine regimen consisting of consecutive priming with DNA and boosting with recombinant fowlpox virus. J Virol. 1998; 72:10180-10188. [PubMed: 9811759] 
21. Shiver JW, Fu TM, Chen L, Casimiro DR, Davies ME, Evans RK, Zhang ZQ, Simon AJ, Trigona WL, Dubey SA, et al. Replication-incompetent adenoviral vaccine vector elicits effective antiimmunodeficiency-virus immunity. Nature. 2002; 415:331-335. [PubMed: 11797011] • Following a report by Amara et al., [18], this is another key study reporting the use of DNA prime-adeno viral vector boost approach was able to elicit protective immune responses against SHIV in a nonhuman primate model.

22. Barouch DH, McKay PF, Sumida SM, Santra S, Jackson SS, Gorgone DA, Lifton MA, Chakrabarti BK, Xu L, Nabel GJ, et al. Plasmid chemokines and colony-stimulating factors enhance the immunogenicity of DNA priming-viral vector boosting human immunodeficiency virus type 1 vaccines. J Virol. 2003; 77:8729-8735. [PubMed: 12885892]

23. Egan MA, Chong SY, Megati S, Montefiori DC, Rose NF, Boyer JD, Sidhu MK, Quiroz J, Rosati M, Schadeck EB, et al. Priming with plasmid DNAs expressing interleukin-12 and simian immunodeficiency virus gag enhances the immunogenicity and efficacy of an experimental AIDS vaccine based on recombinant vesicular stomatitis virus. AIDS Res Hum Retroviruses. 2005; 21:629-643. [PubMed: 16060834]

24. Harari A, Bart PA, Stohr W, Tapia G, Garcia M, Medjitna-Rais E, Burnet S, Cellerai C, Erlwein O, Barber T, et al. An HIV-1 clade C DNA prime, NYVAC boost vaccine regimen induces reliable, polyfunctional, and long-lasting T cell responses. J Exp Med. 2008; 205:63-77. [PubMed: $18195071] \bullet$ Human study results showed that a DNA prime-pox vector boost HIV vaccine elicited higher level and better quality of cell mediated immune responses in humans when compared to a pox vector only HIV vaccine with the same antigens in both vaccines.

25. Nitayaphan S, Pitisuttithum P, Karnasuta C, Eamsila C, de Souza M, Morgan P, Polonis V, Benenson M, VanCott T, Ratto-Kim S, et al. Safety and immunogenicity of an HIV subtype B and E prime-boost vaccine combination in HIV-negative Thai adults. J Infect Dis. 2004; 190:702-706. [PubMed: 15272397]

26. Dunachie SJ, Walther M, Epstein JE, Keating S, Berthoud T, Andrews L, Andersen RF, Bejon P, Goonetilleke N, Poulton I, et al. A DNA prime-modified vaccinia virus ankara boost vaccine encoding thrombospondin-related adhesion protein but not circumsporozoite protein partially protects healthy malaria-naive adults against Plasmodium falciparum sporozoite challenge. Infect Immun. 2006; 74:5933-5942. [PubMed: 16988273]

27. Magalhaes I, Sizemore DR, Ahmed RK, Mueller S, Wehlin L, Scanga C, Weichold F, Schirru G, Pau MG, Goudsmit J, et al. rBCG induces strong antigen-specific T cell responses in rhesus macaques in a prime-boost setting with an adenovirus 35 tuberculosis vaccine vector. PLoS ONE. 2008; 3:e3790. [PubMed: 19023426] • Mixing a bacterial vector and a viral vector for eliciting cell medicated immune responses in a non-human primate model.

28. Cai H, Yu DH, Hu XD, Li SX, Zhu YX. A combined DNA vaccine-prime, BCG-boost strategy results in better protection against Mycobacterium bovis challenge. DNA Cell Biol. 2006; 25:438447. [PubMed: 16907641]

29. Wang QM, Sun SH, Hu ZL, Yin M, Xiao CJ, Zhang JC. Improved immunogenicity of a tuberculosis DNA vaccine encoding ESAT6 by DNA priming and protein boosting. Vaccine. 2004; 22:3622-3627. [PubMed: 15315841]

30. Sin JI, Bagarazzi M, Pachuk C, Weiner DB. DNA priming-protein boosting enhances both antigenspecific antibody and Th1-type cellular immune responses in a murine herpes simplex virus-2 gD vaccine model. DNA Cell Biol. 1999; 18:771-779. [PubMed: 10541436]

31. Biswas S, Reddy GS, Srinivasan VA, Rangarajan PN. Preexposure efficacy of a novel combination DNA and inactivated rabies virus vaccine. Hum Gene Ther. 2001; 12:1917-1922. [PubMed: 11589833]

32. Herrmann JE, Wang S, Zhang C, Panchal RG, Bavari S, Lyons CR, Lovchik JA, Golding B, Shiloach J, Lu S. Passive immunotherapy of Bacillus anthracis pulmonary infection in mice with antisera produced by DNA immunization. Vaccine. 2006; 24:5872-5880. [PubMed: 16790303]

33. Xiao-wen H, Shu-han S, Zhen-lin H, Jun L, Lei J, Feng-juan Z, Ya-nan Z, Ying-jun G. Augmented humoral and cellular immune responses of a hepatitis B DNA vaccine encoding HBsAg by protein boosting. Vaccine. 2005; 23:1649-1656. [PubMed: 15705468]

34. Wang S, Parker C, Taaffe J, Solorzano A, Garcia-Sastre A, Lu S. Heterologous HA DNA vaccine prime--inactivated influenza vaccine boost is more effective than using DNA or inactivated 
vaccine alone in eliciting antibody responses against $\mathrm{H} 1$ or $\mathrm{H} 3$ serotype influenza viruses. Vaccine. 2008; 26:3626-3633. [PubMed: 18538900] •• Heterologous DNA prime-inactivated flu vaccine boost is more effective than the homologous prime-boost with inactivated flu vaccine in eliciting protective antibody responses in an animal model naïve to flu antigen, pointing to new vaccination strategies against influenza viruses that may cause pandemic flu in the world human populations.

35. Garcia-Hernandez Mde L, Gray A, Hubby B, Kast WM. In vivo effects of vaccination with sixtransmembrane epithelial antigen of the prostate: a candidate antigen for treating prostate cancer. Cancer Res. 2007; 67:1344-1351. [PubMed: 17283172]

36. Skinner MA, Wedlock DN, de Lisle GW, Cooke MM, Tascon RE, Ferraz JC, Lowrie DB, Vordermeier HM, Hewinson RG, Buddle BM. The order of prime-boost vaccination of neonatal calves with Mycobacterium bovis BCG and a DNA vaccine encoding mycobacterial proteins Hsp65, Hsp70, and Apa is not critical for enhancing protection against bovine tuberculosis. Infect Immun. 2005; 73:4441-4444. [PubMed: 15972546]

37. Park SH, Yang SH, Lee CG, Youn JW, Chang J, Sung YC. Efficient induction of T helper 1 CD4+ T-cell responses to hepatitis $\mathrm{C}$ virus core and $\mathrm{E} 2$ by a DNA prime-adenovirus boost. Vaccine. 2003; 21:4555-4564. [PubMed: 14575768] • The order of prime-boost with DNA and adenovirus vector vaccines is important for the induction of cell mediated immune responses against HCV E2 antigen.

38. Vaine M, Wang S, Crooks ET, Jiang P, Montefiori DC, Binley J, Lu S. Improved induction of antibodies against key neutralizing epitopes by human immunodeficiency virus type 1 gp120 DNA prime-protein boost vaccination compared to gp120 protein-only vaccination. J Virol. 2008; 82:7369-7378. [PubMed: 18495775] • Including a DNA priming immunization was able to elicit conformation sensitive antibody responses when compared to protein alone HIV-1 Env vaccine.

39. Otten GR, Schaefer M, Doe B, Liu H, Srivastava I, Megede J, Kazzaz J, Lian Y, Singh M, Ugozzoli M, et al. Enhanced potency of plasmid DNA microparticle human immunodeficiency virus vaccines in rhesus macaques by using a priming-boosting regimen with recombinant proteins. J Virol. 2005; 79:8189-8200. [PubMed: 15956564] 
Table 1

Common Heterologous Prime-Boost Vaccinations

\begin{tabular}{ccc}
\hline Prime immunization & Boost immunization & Representative references \\
\hline DNA & Recombinant protein & {$[14,17]$} \\
\hline & Inactivated Vaccine & {$[31,34]$} \\
\hline & Viral vectors & {$[19-21,23,24]$} \\
\hline Biral vector & Recombinant Protein & {$[36]$} \\
\hline BCG & Viral vector & {$[4]$} \\
\hline
\end{tabular}

